\title{
Towards Integration of Statistical Hypothesis Tests into Deep Neural Networks
}

\author{
Ahmad Aghaebrahimian \\ Zurich University of Applied Sciences \\ Switzerland \\ agha@zhaw.ch
}

\author{
Mark Cieliebak \\ Zurich University of Applied Sciences \\ Switzerland \\ cielezhaw.ch
}

\begin{abstract}
We report our ongoing work about a new deep architecture working in tandem with a statistical test procedure for jointly training texts and their label descriptions for multi-label and multi-class classification tasks. A statistical hypothesis testing method is used to extract the most informative words for each given class. These words are used as a class description for more label-aware text classification. Intuition is to help the model to concentrate on more informative words rather than more frequent ones. The model leverages the use of label descriptions in addition to the input text to enhance text classification performance. Our method is entirely data-driven, has no dependency on other sources of information than the training data, and is adaptable to different classification problems by providing appropriate training data without major hyper-parameter tuning. We trained and tested our system on several publicly available datasets, where we managed to improve the state-of-the-art on one set with a high margin, and to obtain competitive results on all other ones.
\end{abstract}

\section{Introduction}

Text classification is a complex problem in Natural Language Processing (NLP) with lots of applications from sentiment analysis (Liu, 2015) to question answering (Aghaebrahimian and Jurčíček, 2016b,a; Yu et al., 2014) or abusive language detection (von Grünigen et al., 2018; Founta et al., 2018), to name just a few. Text classification is defined as the task of assigning a certain pre-defined class to a document.

The number of classes can be arbitrarily large in multi-class classification, whereas there are only two classes for binary classification. In multilabel classification, the number of labels attached to each document is not known and usually larger than one, while in multi-class classification, only one class is assigned to each document.

There exist numerous approaches for text classification, ranging from simple hand-crafted lexicallevel features with Naive Bayes or Support Vector Machines (SVM) (Wang and Manning, 2012) to self-learning approaches with Deep Neural Networks (DNN) (Deriu and Cieliebak, 2017).

For the latter, several architectures such as Convolutional or Recurrent Neural Networks (CNN or RNN) (Shen et al., 2017; Wang et al., 2018b) have been proposed. These architectures learn different levels of textual representation in their layers, which are an essential source of information for the classification process. As an alternative, attention networks are also introduced (Bahdanau et al., 2015; Yang et al., 2016) to capture the features with the highest discriminative power regarding the class and irrespective of their distance. On the other hand, the field of Statistics has since long developed and optimized various methods to capture 'relevant' properties of a given dataset. In this work, we extend DNNs with statistical hypothesis testing methods to enhance their performance in assessing feature relevancy on the input data.

More precisely, our approach works as follows:

- For each class, we generate a class description, which is a set of 'most informative words' that will help to distinguish the class from others.

- To achieve this, we apply two statistical hypothesis testing approaches called $\chi^{2}$ test (Pennington et al., 1893) and Analysis of Variance test (ANOVA) (Fisher, 1921).

- We then extend a DNN that is based on bidirectional Gated Recurrent Units (GRU) with an additional input channel for encoding the class descriptions. This channel uses attention, in addition, to enable the network to focus on the most informative words for each document and given each class. 
Our experiments on four standard datasets show that this approach can already reach or even outperform state-of-the-art solutions for these datasets. While this is very promising, we want to stress already here that this is ongoing work, and that it needs extensive further experiments to fully understand when and why the proposed method works.

The main contributions of this work are the use of statistical hypothesis testing methods specifically for class descriptor extraction rather than feature extraction, and a new deep architecture working in tandem with a statistical test procedure with state-of-the-art performance in multilabel and multi-class classification.

We organize the remaining content into the following sections. After a review on state of the art in Section 2, we describe how we extract the class descriptors in Section 3. Then we continue with a description of our deep architecture, followed by the system setup for our experiments in Sections 4 and 5, respectively. Finally we report our results in Section 6 and conclude in Section 7.

\section{Related Work}

Many solutions for text classification with DNNs use word embeddings as the primary and only representation of the input text. Word embeddings are low-dimensional word vectors that can be precomputed using, for instance, Word2Vec (Mikolov et al., 2013), GloVe (Pennington et al., 2014), or fastText (Bojanowski et al., 2017). Many studies show the importance of embeddings in text classification (Arora et al., 2017; Wieting et al., 2016), and DNNs have been proven very useful in capturing syntactic- and semantic-level textual information. Still, it has been shown that they can benefit from additional representations of the input, e.g., by using a method called Attention Mechanism (Bahdanau et al., 2015; Yang et al., 2016; Cui et al., 2017; Gehring et al., 2017).

The main idea in the attention mechanism for text classification is to put emphasis on more informative tokens for each class. The attention mechanism has been successfully applied for different tasks in NLP, including but not limited to sentiment analysis (Zhou et al., 2016), modeling sentence pair (Aghaebrahimian, 2018a; Yin et al., 2016), question answering (Aghaebrahimian, 2018b; Seo et al., 2016), and summarization (Rush et al., 2015).
The idea of joint learning of text and class descriptions is already practiced by Wang et al. (2018a). They showed that training an attention model on class descriptions in a joint embedding space is beneficial for text classification. However, they extracted class descriptions only from the class names, which limits the functionality of this approach, since in many use cases the names are very short, sometimes even just one token.

In our work, the class descriptions are extracted using a statistical, data-driven approach. This makes our model independent from the label-set description which is not always available.

Our model is also similar to the one by Founta et al. (2018) with two differences. First, they assume that classes are provided with metadata, while our model extracts class descriptions directly from the training data. Second, the token channel and class description channel in our model both have the same time complexity thus they both converge simultaneously, and we do not need to worry about over-fitting one channel while the other is still training.

The use of statistical tests for extracting textual features in text classification is quite common and already proven beneficial. Bahassine et al. (2018), for instance, used three different statistical tests for feature extraction to improve Arabic text classification. However, we do not use statistical tests for feature extraction. Instead, we use them to extract class descriptions which are used as a second channel alongside with their accompanying texts in a deep neural network.

This is the first time that statistical tests are used for extracting class descriptor tokens which can be used for jointly training deep neural models on texts with their class descriptions.

\section{Generating Class Descriptions}

We show how to extract class descriptions using a data-driven method applied to the training data. To retrieve the class descriptions, we use two statistical hypothesis testing approaches called $\chi^{2}$ and Analysis of Variance (ANOVA) tests.

We assume that each class is triggered given specific tokens; hence, given each class, the frequencies of those tokens should be distinguishable from other non-triggering ones. Therefore, for each class, we formulate a null hypothesis (i.e., an indirect assumption) that states that the presence of specific tokens does not have any impact on 
their class determination. Then we check which words can reject the hypothesis, hence, have discriminative significance in distinguishing classes from each other.

The $\chi^{2}$ test is used to determine whether there is a significant difference between the expected frequencies and the observed frequencies of tokens in each one of the classes. Using the training data, we separate the documents into mutually exclusive classes. Given each class and the null hypothesis, we compute the $\chi^{2}$ of the documents' words, which provides us with the probability with which any token falls into the corresponding class.

The $\chi^{2}$ test allows us to evaluate how likely it is to observe a word in a class, assuming the null hypothesis is true.

Similarly, we use the ANOVA F-statistics to determine whether there is a dependence between certain tokens and a specific class, so to eliminate the words that are most likely independent of the class, hence, irrelevant for classification.

Both tests provide each word with a probability given each class. To get an $n$-dimensional class description vector, we extract the $n$ top-rated words for each class and use them as class descriptors. One should be careful not to confuse word embedding dimensions with the dimension of class descriptions. By class description dimension, we mean the length of the string containing the most informative words given each class.

Some of the most informative words given the classes available in the AG News datasets (Del Corso et al., 2005) are presented in Table 1.

\begin{tabular}{|l|l|}
\hline Class & Informative words \\
\hline World & $\begin{array}{l}\text { iraq, minister, president, } \\
\text { prime, baghdad, iraqi, dig, } \\
\text { palestinian, military, nu- } \\
\text { clear, israeli, ... }\end{array}$ \\
\hline Sports & $\begin{array}{l}\text { dig, season, league, team, } \\
\text { game, cup, night, coach, } \\
\text { victory, win, sports, cham- } \\
\text { pionship, olympic, ... }\end{array}$ \\
\hline Business & $\begin{array}{l}\text { oil, stocks, prices, percent, } \\
\text { quickinfo, target, profit, } \\
\text { company, shares, bilion, } \\
\text { quarter, sales, earnings, ... }\end{array}$ \\
\hline Science & $\begin{array}{l}\text { microsoft, software, inter- } \\
\text { net, space, music, com- } \\
\text { puter, users, web, search, } \\
\text { windows, technology, ... }\end{array}$ \\
\hline
\end{tabular}

Table 1: Extracted $\chi^{2}$ words for the AG News dataset

\section{Model}

The overall architecture of our system is illustrated in Figure 1. We use a lookup table for transforming token indices to embedding vectors. The embeddings are fed into a bidirectional Gated Recurrent Unit (BiGRU) (Cho et al., 2014). The resulting tensors are then max- and average-pooled to extract the most relevant features. At the end of this channel, we have the vector of an encoded text.

A similar channel is used for encoding the class descriptions. Using the words extracted by the $\chi^{2}$ test as described in Section 3, we generate a new string in which only the $\chi^{2}$ words are available. Given each class, this contains the highest informative words for this class. Additionally, we put an attention layer on top of this channel to learn the importance of each word given a particular class.

The attention layer is implemented based on the work of Yang et al. (2016). The mathematical representation is as follow:

$$
\begin{aligned}
u & =f(\omega \cdot h+b) \\
a_{i} & =\operatorname{Softmax}\left(u_{i} \cdot u_{s}\right) \\
v_{i} & =\sigma_{i} a_{i} \cdot h_{i} .
\end{aligned}
$$

where $h$ are the tensors out of the BiGRU layer, and $w, b, a$, and $v$ are the weight vectors, bias terms, attention vectors, and document vectors respectively.

Finally, we concatenate the resulting tensors from the attention layer with the max- and average-pooling layers and feed them into a dense layer for final classification.

For multi-class classification it is common to use the Softmax function

$$
P\left(c_{j} \mid x_{i}\right)=\frac{\exp \left(\omega_{j} \cdot x_{i}\right)}{\sum_{c=1}^{C} \exp \left(\omega_{c} \cdot x_{i}\right)} .
$$

where $x_{i}, c$, and $\omega$ are features, classes, and associated weight vectors, respectively. This function is used as the dense output layer of the network to normalize the logits generated by previous layers. In this way, we can model the probability of class $c_{j}$ as a multi-nominal distribution. The consequence of this decision is that the probability for a class is not independent of the other class probabilities, which would not be the desired behavior when dealing with a multi-label classification task. For instance, in a multi-label classification for hate speech detection, the probability of a 


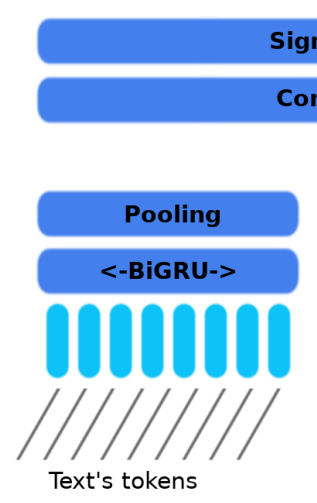

Figure 1: The system architecture

comment for being offensive is independent of its probability of being hateful, because an offensive tone can be used in a text that is not necessarily hateful (Founta et al., 2018). For this reason, instead of Softmax, we use the Sigmoid activation function

$$
\sigma(z)=\frac{1}{1+\exp (-z)}
$$

which is a better choice for multi-label classification. In this way we can model the probability of a class as Bernoulli's distribution

$$
P\left(c_{j} \mid x_{i}\right)=\frac{1}{1+\exp \left(-\omega_{j} \cdot x_{i}\right)}
$$

which makes the probability of each class $c_{j}$ independent from the other class probabilities.

Therefore we use a Softmax dense layer for multi-class and Sigmoid dense layer for multilabel classification to get the probabilities associated with each target class.

\section{System Setup}

For the text representation in our system, we use pre-trained Glove embeddings (Pennington et al., 2014) trained on 840 billion tokens with 300dimensional vectors and set it to get updated through training. As the loss function for multiclass and multi-label settings, we use the Categorical and the Binary cross-entropy, respectively. We define the bidirectional GRUs, each with 128 units. We also set both the drop-outs (Srivastava et al., 2014) and recurrent drop-outs to 0.5. In the following subsections, some details concerning the pre-processing of tested datasets are presented.

\subsection{Multi-label}

Data. For the multi-label classification task, we train and test our model on a large publicly available dataset provided for toxic comment classification in a Kaggle competition called 'Toxic Comment Classification Challenge.' The texts of the dataset were extracted from Wikipedia comments and have been labeled by human raters for six categories of toxic behavior: toxic, severe-toxic, obscene, threat, insult, and identity-hate. The training and test datasets contain $160 \mathrm{k}$ and $153 \mathrm{k}$ comments, respectively. The task is to train a model which assigns a probability to each of the six categories of toxic behavior given a new comment.

Pre-processing. The pre-processing step for this dataset is performed by lower-casing, cleaning the comments from non-alphanumeric characters, using the first $130 \mathrm{k}$ most frequent tokens and removing comments longer than 80 tokens (95\% percentile of the training dataset). Shorter comments are padded with zero to fixate the length of all comments to 80 tokens.

Performance Measure. The Area Under the Receiver Operating Characteristic Curve (AUCROC) is used to measure the performance of the systems. ROC is a probability curve, and AUC is a measure of separability. This measure tells how much a model is capable of distinguishing between classes.

Since the output of the model is a vector of probabilities that the model computes for each class and we want to assign more than one class to each text, we define a threshold using the validation data and accept all the classes with probabilities above the threshold as positive class.

\subsection{Multi-class}

Data. We also train and test our system on three other datasets for multi-class classifications, namely Hate Speech dataset (Davidson et al., 2017), AG News (Del Corso et al., 2005), and DBpedia, to measure its performance on multi-class classification. Some statistics of these datasets are reported in Table 2.

\begin{tabular}{|l|c|c|c|c|}
\hline Dataset & Type & Classes/Labels & Training & Testing \\
\hline Hate Speech & Multi-class & 3 & $22.5 \mathrm{~K}$ & $2.5 \mathrm{~K}$ \\
\hline DBpedia & Multi-class & 14 & $560 \mathrm{~K}$ & $70 \mathrm{~K}$ \\
\hline AG News & Multi-class & 4 & $120 \mathrm{~K}$ & $7.6 \mathrm{~K}$ \\
\hline Kaggle-toxic comments & Multi-label & 6 & $160 \mathrm{~K}$ & $153 \mathrm{~K}$ \\
\hline
\end{tabular}

Table 2: Types, number of classes, and number of training/testing samples in the datasets used for training in this work 
Pre-processing. The pre-processing step for these datasets is performed by lower-casing, removing non-alphanumeric characters, and removing repetitive characters from tokens (e.g. yoooouuuuu $->$ you).

Performance Measure. In contrast to the multi-label setting, in the multi-class setting, we do not need to define a threshold. Instead, we get the argmax of the vector of probabilities since we need to return only one class.

\section{Experimental Results}

Table 3 shows that the system obtains superior results in the Hate Speech dataset and yields competitive results on the Kaggle data in comparison to some sate-of-the-art baseline systems. Table 4 shows the results of our system on the DBpedia and AG News datasets. Using the same model without any tuning, we managed to obtain competitive results again compared to previous stateof-the-art systems.

We also ran preliminary experiments on class description vectors with different dimensions (50 vs. 100), indicated by the suffix of each name in Table 3. By dimension, we mean the number of words given each label and not the dimension of word vectors which are all the same for both channels (i.e., 300).

It turns out that in all but one case, the more words, the better the performance. However, we did not get statistically significant results with class descriptors with dimensions higher than 100. It seems that the range 50-100 is the optimal dimension for this approach and these datasets. Bigger vectors such as 150 did not yield any statistically significant improvement in performance, and 200-, and 300-dimensional vectors deteriorated the performance.

We observed that the decline in the performance comes mainly from two sources: the network over-fit, and the similar words in different classes. By increasing the number of informative words, the number of similar words in different classes increases which leads to sub-optimal classification decision boundaries.

\section{Conclusion}

Previous studies in text classification have shown that training classifiers with class descriptions or class metadata alongside the text is beneficial. However, many of these systems depend on the

\begin{tabular}{|l|c|c|c|c|}
\hline Hate Speech dataset & $\mathrm{P}(\%)$ & $\mathrm{R}(\%)$ & $\mathrm{F} 1(\%)$ & $\mathrm{AUC}(\%)$ \\
\hline (Davidson et al., 2017) & 91 & 90 & 90 & 87 \\
\hline (Founta et al., 2018) & 89 & 89 & 89 & 92 \\
\hline This work+ $\chi^{2} 50$ & 89.7 & 90.4 & 90 & 92.9 \\
\hline This work+ $\chi^{2} 100$ & 90.3 & $\mathbf{9 2 . 5}$ & $\mathbf{9 1 . 3}$ & $\mathbf{9 3 . 7}$ \\
\hline This work+ANOVA50 & 89.2 & 89.6 & 89.3 & 92.1 \\
\hline This work+ANOVA100 & 89.8 & 89.2 & 89.4 & 92.4 \\
\hline Kaggle dataset & & & & \\
\hline Leader-board & - & - & - & 98.82 \\
\hline This work+ $\chi^{2} 50$ & - & - & - & 98.05 \\
\hline This work $+\chi^{2} 100$ & - & - & - & 98.24 \\
\hline
\end{tabular}

Table 3: The results of our system on the Hate Speech and Kaggle datasets. With one exception, in all cases longer class description leads to better performance. The results of the Kaggle dataset are only reported in AUC to be comparable with other systems in the multilabel category.

\begin{tabular}{|l|c|c|}
\hline & DBpedia(\%) & AG News(\%) \\
\hline Bi-BloSAN(Shen et al., 2018) & 98.77 & 93.32 \\
\hline LEAM(Wang et al., 2018a) & 99.02 & 92.45 \\
\hline This work & 98.90 & 92.05 \\
\hline
\end{tabular}

Table 4: Competitive results on DBpedia and AG News reported in accuracy (\%) without any hyper-parameter tuning.

provided label set for generating their class descriptors. This dependence on an external source of information limits their applicability when such information is not available.

In this paper, we proposed a data-driven approach for extracting class descriptions for jointly training text with their class descriptors, based on pure statistical tests. Moreover, we designed a new deep neural architecture to make use of the output of this statistical approach for enhancing the performance of text classification by attending on the informative words of each class. Although we have shown that the approach works in principle, by achieving state-of-the-art results on four standard datasets, it needs to be further explored in order to understand why it works. In particular, we need to understand why words extracted with $\chi^{2}$ yield better results compared to ANOVA, how many words should be extracted given a specific task, if other statistical tests might even improve the outcomes, etc. Once this understanding is achieved, this may lead us towards proposing better data-driven approaches for extracting class descriptions that will be beneficial in text classification. 


\section{References}

Ahmad Aghaebrahimian. 2018a. Deep neural networks at the service of multilingual parallel sentence extraction. In Proceedings of the 27th International Conference on Computational Linguistics (CoLing), pages 1372-1383, Santa Fe, New Mexico, USA. Association for Computational Linguistics.

Ahmad Aghaebrahimian. 2018b. Linguistically-based deep unstructured question answering. In Proceedings of the 22nd Conference on Computational Natural Language Learning (CoNLL), pages 433-443, Brussels, Belgium. Association for Computational Linguistics.

Ahmad Aghaebrahimian and Filip Jurčíček. 2016a. Constraint-based open-domain question answering using knowledge graph search. In Proceedings of the 19th International Conference of Text, Speech, and Dialogue (TSD), volume 9924, pages 28-36.

Ahmad Aghaebrahimian and Filip Jurčíček. 2016b. Open-domain factoid question answering via knowledge graph search. In Proceedings of the Conference of the North American Chapter of the Association for Computational Linguistics (NAACL)- the Workshop on Human-Computer Question Answering, pages 22-28, San Diego, California. Association for Computational Linguistics.

Sanjeev Arora, Yingyu Liang, and Tengyu Ma. 2017. A simple but tough-to-beat baseline for sentence embeddings. In Proceedings of the International Conference on Learning Representations (ICLR).

Said Bahassine, Abdellah Madani, Mohammed AlSarem, and Mohamed Kissi. 2018. Feature selection using an improved chi-square for arabic text classification. Journal of King Saud University - Computer and Information Sciences.

Dzmitry Bahdanau, Kyunghyun Cho, and Yoshua Bengio. 2015. Neural machine translation by jointly learning to align and translate. In Proceedings of the International Conference on Learning Representations (ICLR).

Piotr Bojanowski, Edouard Grave, Armand Joulin, and Tomas Mikolov. 2017. Enriching word vectors with subword information. Transactions of the Association for Computational Linguistics, 5:135-146.

Kyunghyun Cho, Bart van Merrienboer, Dzmitry Bahdanau, and Yoshua Bengio. 2014. On the properties of neural machine translation: Encoder-decoder approaches. In Proceedings of the Eighth Workshop on Syntax, Semantics and Structure in Statistical Translation.

Yiming Cui, Zhipeng Chen, Si Wei, Shijin Wang, Ting Liu, and Guoping Hu. 2017. Attention-overattention neural networks for reading comprehension. In Proceedings of the 55th Annual Meeting of the Association for Computational Linguistics (Volume 1: Long Papers), pages 593-602, Vancouver, Canada. Association for Computational Linguistics.
Thomas Davidson, Dana Warmsley, Michael W. Macy, and Ingmar Weber. 2017. Automated hate speech detection and the problem of offensive language. In Proceedings of the International AAAI Conference on Web and Social Media (ICWSM).

Gianna M. Del Corso, Antonio Gullí, and Francesco Romani. 2005. Ranking a stream of news. In Proceedings of the 14th International Conference on World Wide Web, WWW'05, pages 97-106, New York, NY, USA. ACM.

Jan Milan Deriu and Mark Cieliebak. 2017. Swissalps at semeval-2017 task 3 : attention-based convolutional neural network for community question answering. In Proceedings of the 11th International Workshop on Semantic Evaluation (SemEval-2017), pages 334-338, Canberra, Australia. Association for Computational Linguistics.

Ronald A. Fisher. 1921. On the 'probable error' of a coefficient of correlation deduced from a small sample. Metron, 1:3-32.

Antigoni-Maria Founta, Despoina Chatzakou, Nicolas Kourtellis, Jeremy Blackburn, Athena Vakali, and Ilias Leontiadis. 2018. A unified deep learning architecture for abuse detection. Computing Research Repository, CoRR, abs/1802.00385.

Jonas Gehring, Michael Auli, David Grangier, Denis Yarats, and Yann N Dauphin. 2017. Convolutional sequence to sequence learning. arXiv preprint, arXiv:1705.03122.

Dirk von Grünigen, Ralf Grubenmann, Fernando Benites, Pius Von Däniken, and Mark Cieliebak. 2018. spMMMP at GermEval 2018 shared task: Classification of offensive content in tweets using convolutional neural networks and gated recurrent units. In Proceedings of the GermEval 2018 Workshop : 14th Conference on Natural Language Processing (KONVENS).

Bing Liu. 2015. Sentiment Analysis. Cambridge University Press, Cambridge, UK.

Tomas Mikolov, Kai Chen, Greg Corrado, and Jeffrey Dean. 2013. Efficient estimation of word representations in vector space. arXiv:1301.3781.

Jeffrey Pennington, Richard Socher, and Christopher Manning. 1893. Contributions to the mathematical theory of evolution. In Proceedings of the Royal Society.

Jeffrey Pennington, Richard Socher, and Christopher Manning. 2014. Glove: Global vectors for word representation. In Proceedings of the Conference on Empirical Methods in Natural Language Processing (EMNLP).

Alexander M. Rush, Sumit Chopra, and Jason Weston. 2015. A neural attention model for abstractive sentence summarization. In Proceedings of the 2015 
Conference on Empirical Methods in Natural Language Processing, pages 379-389, Lisbon, Portugal. Association for Computational Linguistics.

Minjoon Seo, Aniruddha Kembhavi, Ali Farhadi, and Hannaneh Hajishirzi. 2016. Bidirectional attention flow for machine comprehension. arXiv:1611.01603.

Dinghan Shen, Yizhe Zhang, Ricardo Henao, Qinliang $\mathrm{Su}$, and Lawrence Carin. 2017. Deconvolutional latent-variable model for text sequence matching. CoRR, abs/1709.07109.

Tao Shen, Tianyi Zhou, Guodong Long, Jing Jiang, and Chengqi Zhang. 2018. Bi-directional block selfattention for fast and memory-efficient sequence modeling. In Proceedings of the International Conference on Learning Representations (ICLR).

Nitish Srivastava, Geoffrey Hinton, Alex Krizhevsky, Ilya Sutskever, and Ruslan Salakhutdinov. 2014. Dropout: A simple way to prevent neural networks from over fitting. Journal of Machine Learning Research, 15(1):1929-1958.

Guoyin Wang, Chunyuan Li, Wenlin Wang, Yizhe Zhang, Dinghan Shen, Xinyuan Zhang, Ricardo Henao, and Lawrence Carin. 2018a. Joint embedding of words and labels for text classification. In Proceedings of the Association for Computational Linguistics (ACL).

Sida Wang and Christopher D Manning. 2012. Baselines and bigrams: Simple, good sentiment and topic classification. In Proceedings of the Association for Computational Linguistics (ACL).

Wenlin Wang, Zhe Gan, Wenqi Wang, Dinghan Shen, Jiaji Huang, Wei Ping, Sanjeev Satheesh, and Lawrence Carin. 2018b. Topic compositional neural language model. In Proceedings of the International Conference on Artificial Intelligence and Statistics (AISTATS).

John Wieting, Mohit Bansal, Kevin Gimpel, and Karen Livescu. 2016. Towards universal paraphrastic sentence embeddings. In Proceedings of the International Conference on Learning Representations (ICLR).

Zichao Yang, Diyi Yang, Chris Dyer, Xiaodong He, Alex Smola, and Eduard Hovy. 2016. Hierarchical attention networks for document classification. In Proceedings of the Conference of the North American Chapter of the Association for Computational Linguistics (NAACL).

Wenpeng Yin, Hinrich Schutze, Bing Xiang, and Bowen Zhou. 2016. Abcnn: Attention-based convolutional neural network for modeling sentence pairs. Transactions of the Association for Computational Linguistics (TACL).
Lei Yu, Karl Moritz Hermann, Phil Blunsom, and Stephen Pulman. 2014. Deep learning for answer sentence selection. In Proceedings of the Conference on Neural Information Processing Systems (NIPS) - Deep learning workshop.

Xinjie Zhou, Xiaojun Wan, , and Jianguo Xiao. 2016. Attention-based lstm network for cross-lingual sentiment classification. In Proceedings of the conference on Empirical Methods in Natural Language Processing (EMNLP). 